\title{
45. THE SPACE DISTRIBUTION OF THE OB-TYPE STARS IN CARINA
}

\author{
J. A. G R AHA M \\ Cerro Tololo Inter-American Observatory, La Serena, Chile*
}

\begin{abstract}
Distances have been determined for 436 OB-type stars in Carina. A sharp outside edge is found to the OB star distribution with respect to the center of the Galaxy. The layer of OB stars follows the galactic plane to distances of the order of $3 \mathrm{kpc}$, but at distances greater than $4 \mathrm{kpc}$ it appears to bend away from the plane to negative latitudes by $2^{\circ}$ or $3^{\circ}$ out to distances of the order of $10 \mathrm{kpc}$.
\end{abstract}

The Carina section of the Milky Way is at present perhaps the most promising of all fields for the optical study of a single large scale feature of the Galaxy. An increasing amount of evidence confirms that the very many young stars of population I are spread out over distances of several kiloparsecs along the line of sight. Young open clusters and associations, Hil regions, OB-type stars, cepheid variables and WolfRayet stars are all found here over a very wide range of apparent magnitude. On the other hand, the interstellar absorption is not, on the average, very heavy and it does not appear to increase rapidly with distance. This contrasts with the situation in most other directions in the galactic plane where there are strong concentrations of population I and it is this characteristic more than any other which allows us to study the Carina region optically over great distances with the presently available observational techniques.

In this short report, the preliminary results of a study of the OB star distribution are reported. The observing list was the objective prism survey made in this part of the sky by Graham and Lyngå (1965). In this publication 454 stars of type OB are listed. They are mainly stars with apparent magnitudes between 8.5 and 11.5 and are spread over an area of approximately 75 sq. deg. centered on the star $\eta$ Carinae. Because of crowding problems, the stars contained in several young open clusters are not included in the Graham-Lyngå survey. Magnitudes and colors for the OB survey stars have now been published. These were measured with the Leiden Observatory's 5-color photoelectric photometer using the system of Walraven and Walraven (1960). On the basis of the intrinsic color relations published by Walraven (1966), these data have been used to derive absorption corrected visual magnitudes. A ratio of total to selective absorption corresponding to 3.2 on the UBV system is assumed. Absolute magnitudes are derived mainly from the $\mathrm{H} \beta$ photometry which has recently been obtained at Cerro Tololo Inter-American Observatory and is now being prepared for publication. The calibration relation for converting the observed $\mathrm{H} \beta$ indices into absolute magnitudes is the mean of the two relations given by Fernie (1965) and by Graham (1967). For the spectral type OB, there is good agreement with the new calibration

* Operated by the Association of Universities for Research in Astronomy, Inc., under contract with the National Science Foundation. 
relation reported at this symposium by Crawford. Using the absolute magnitudes derived in this way, distance moduli and distances are then computed. The main advantage of the $\mathrm{H} \beta$ method lies in the simple yet efficient technique used to obtain a limited amount of information for a large number of stars. By nature of its derivation, the $\mathrm{H} \beta$ index is independent of both atmospheric and interstellar reddening. The observation and reduction procedure is very straightforward although the uncertainties of the absolute magnitudes do place a limit on the utility of the method. The contributors to the scatter in absolute magnitude include $\mathrm{H} \beta$ line emission (even though the $\mathrm{H} \delta$ surveys of The (1966) and Wray (1967) identify nearly all the Be type stars in the field) as well as stellar multiplicity rotation and evolutionary effects. It is difficult to see how the uncertainty of an absolute magnitude derived in this way can be appreciably reduced from its present value of about 0.6 magnitudes (standard error) without a detailed study of every program star to assess the contribution of each of the above effects. A full discussion of these errors will be given in the paper now in preparation.

In the preliminary study of the overall distribution of the present sample of $O B$ stars, two diagrams have been made. Distance is plotted in the first diagram against galactic longitude and in the second diagram against distance, $z$, from the new galactic plane. The longitude (new) range of the OB star survey is $282^{\circ}$ to $292^{\circ}$ and the many stars with distances of the order of $2 \mathrm{kpc}$ are fairly evenly distributed over the whole longitude range. On the other hand, the stars with distances greater than $5 \mathrm{kpc}$ are only found in the longitude sector $285^{\circ}$ to $292^{\circ}$. The simplest and most likely interpretation of the results is that we are seeing a real outside edge to the OB star concentration in this direction. The edge is apparently in the direction $287^{\circ}$. There is a suggestion that a tangential point may be observed at longitude $283^{\circ} .5$ distance $1.8 \mathrm{kpc}$. This result is still uncertain and needs confirmation. There appears to be a less certain extension of this edge out to $10 \mathrm{kpc}$. Both $\mathrm{H} \beta$ photometry and spectra agree in showing that several of the stars which have been observed must have distances of this order. We seem to be slightly inside this edge and therefore its distance from the sun varies with galactic longitude. If, as seems likely, the normal absorption law is valid, then there is the following relation between galactic longitude and the distance to the edge:

$\begin{array}{ll}l^{\text {II }} & \mathrm{d}(\mathrm{kpc}) \\ 283.5 & 1.8 \\ 284.0 & 2.0 \\ 284.5 & 4.0 \\ 285.0 & 5.0 \\ 285.5 & 6.0\end{array}$

With the second diagram, in which the distance, $z$, from the galactic plane is plotted against the distance from the sun for each star, a number of conclusions can be drawn about the distribution of the stars perpendicular to the galactic plane. At distances less than $4 \mathrm{kpc}$, the stars scatter more or less symmetrically about the galactic plane with a dispersion $|z|$ of about $40 \mathrm{pc}$. The $z$ dispersion of the necessarily young 
sample of supergiant stars is significantly less than the $z$ dispersion of the sample of less luminous stars which contains stars several times as old. At distances greater than $4 \mathrm{kpc}$ the data are consistent with a general bending or distortion of the galactic plane by $2^{\circ}$ or $3^{\circ}$ to negative latitudes out to distances of the order of $10 \mathrm{kpc}$. In the region between longitudes $288^{\circ}$ and $291^{\circ}$ there is some support for this picture from the $21 \mathrm{~cm} \mathrm{HI}$ data presented by Kerr at this symposium. Plans are now being made to obtain radial velocities for a sample of these distant stars in order to make detailed comparisons with the velocity of the neutral hydrogen in this part of the sky.

\section{References}

Fernie, J. D.: 1965, Astron. J. 70, 575.

Graham, J. A.: 1967, Monthly Notices Roy. Astron. Soc. 135, 377.

Graham, J. A. and Lyngå, G.: 1965, Mem. Mt Stromlo Obs. 4, 16.

The, Pik-Sin: 1966, Contr. Bosscha Obs., 35.

Walraven, T.: 1966, IAU Symposium No. 24, p. 274.

Walraven, T. and Walraven, J. H.: 1960, Bull. Astron. Inst. Netherl. 15, 67.

Wray, J. R.: 1967, Ph.D. Thesis, Northwestern University. 